\title{
Test performance and predictors of accuracy of endoscopic ultrasound-guided fine-needle aspiration for diagnosing biliary strictures or masses
}

\section{다(1)우우}

\author{
Authors \\ Tim Raine1, John P. Thomas², Rebecca Brais ${ }^{3}$, Edmund Godfrey ${ }^{4}$, Nicholas R. Carroll ${ }^{4}$, Andrew J. Metz ${ }^{5}$
}

Institutions

1 Division of Gastroenterology, Department of Medicine, Addenbrooke's Hospital, University of Cambridge, Cambridge, UK

2 Department of Gastroenterology, Norfolk and Norwich University Hospital, Norwich, UK

3 Department of Histopathology, Cambridge University Hospitals NHS Foundation Trust, Cambridge, UK

4 Department of Endoscopy, Cambridge University Hospitals NHS Foundation Trust, Cambridge, UK

5 Department of Gastroenterology, Royal Melbourne Hospital, Melbourne, Australia

submitted 2.2.2020

accepted after revision $\quad 5.6 .2020$

Bibliography

Endoscopy International Open 2020; 08: E1537-E1544

DOI 10.1055/a-1231-4948

ISSN 2364-3722

(C) 2020. The Author(s).

This is an open access article published by Thieme under the terms of the Creative Commons Attribution-NonDerivative-NonCommercial License, permitting copying and reproduction so long as the original work is given appropriate credit. Contents may not be used for commecial purposes, or adapted, remixed, transformed or built upon. (https://creativecommons.org/licenses/by-nc-nd/4.0/)

Corresponding author

Dr. Andrew J. Metz, Department of Gastroenterology, Royal

Melbourne Hospital, Melbourne, Australia

Fax: +0160328286

Andrew.Metz@mh.org.au

Supplementary material is available under

https://doi.org/10.1055/a-1231-4948

\section{ABSTRACT}

Background and study aims Endoscopic ultrasoundguided fine-needle aspiration (EUS-FNA) has emerged as an important method for obtaining a preoperative tissue diagnosis for suspected cholangiocarcinoma. However, doubts remain about test sensitivity. This study assessed the value and limitations of EUS-FNA in clinical practice.

Patients and methods Patients undergoing EUS-FNA for biliary strictures/masses at a UK tertiary referral center from 2005 to 2014 were prospectively enrolled. Data on EUS-FNA findings, histology, and endoscopy and patient outcomes were collected to evaluate test performance and identify factors predictive of an inaccurate diagnostic result.

Results Ninety-seven patients underwent a total of 112 EUS-FNA procedures. Overall test sensitivity for an initial EUS-FNA for suspected cholangiocarcinoma was 75\% (95\% Cl 64\%-84\%), with specificity $100 \%$ (95\% Cl 85\%-100\%) and negative predictive value 0.62 (95\% Cl 0.47-0.75). Hilar lesions, the presence of a biliary stent, and a diagnosis of PSC were significantly independently associated with an inaccurate result. For the most difficult cases, repeat sampling and use of the Papanicolaou cytopathology grading scale led to an increase in test sensitivity from $17 \%$ to $100 \%(P=0.015)$ with no loss of specificity.

Conclusions EUS-FNA was found to be a useful method for obtaining a preoperative tissue diagnosis for patients with suspected cholangiocarcinoma. This study identified markers that can reduce test accuracy and measures that can improve test performance of EUS-FNA.

\section{Introduction}

Cholangiocarcinomas (CCA) account for around $3 \%$ of gastrointestinal malignancies, although evidence demonstrates that the incidence and mortality is increasing worldwide [1,2]. The typical clinical presentation is of painless jaundice once biliary obstruction has developed. The initial investigation is usually abdominal ultrasound followed by computed tomography (CT) imaging and magnetic resonance cholangiopancreatography 
(MRCP). Raised serum CA 19-9 levels may be detected and increase suspicion of the diagnosis, particularly if very high [3].

It is important to pursue a pretreatment tissue diagnosis. Previous case series have suggested that $13 \%$ to $33 \%$ of those undergoing surgery for suspected CCA, with consistent symptoms and imaging, have a final diagnosis of another condition such as a benign inflammatory stricture, metastasis, hepatocelIular carcinoma or lymphoma. In these cases, a Whipple's procedure or hemi-hepatectomy, with mortality and morbidity rates up to $3 \%$ and $20 \%$ respectively, could have been avoided [4-9]. In addition, accurate tissue sampling is important for patient counseling and management decisions, with an expanding range of treatment options including neo-adjuvant chemotherapy or radiotherapy.

Unfortunately, making a definitive preoperative diagnosis can be difficult. While the specificity of intraductal brushings during endoscopic retrograde cholangiopancreatography (ERCP) is close to $100 \%$, the sensitivity varies between $9 \%$ and $58 \%$ [10-12]. Endoscopic ultrasound-guided fine-needle aspiration (EUS-FNA) has emerged as the most accurate, safe, and cost-effective technique for obtaining tissue and preventing unnecessary operations [13]. However, due to the paucity of published data in limited case series, doubts remain about the sensitivity and negative predictive value (NPV) of EUS-FNA, with a negative result often failing to reassure.

The purpose of this study was to understand the value and limitations of EUS-FNA in clinical practice, in particular in circumstances when no evidence of malignancy is found. We used data from a large cohort of patients undergoing EUS-FNA for structural biliary abnormalities at a major centralized UK referral service, to determine clinical factors predictive of test performance, when compared with resection specimens or adequate clinical follow-up. In addition, we assessed the value of repeated procedures in excluding cancer, and evaluated the utility of cytological scoring systems in clinical practice. In so doing, we aimed to describe clinical and cytological factors that allow confidence in use of EUS-FNA, with the aim of avoiding unnecessary, highly morbid surgery.

\section{Patients and methods}

\section{Inclusion and exclusion criteria}

The inclusion criteria for this study were all patients in the East of England region (population 5.85 million) who were referred for EUS-FNA following review of radiological imaging suggestive of a biliary mass or stricture at a dedicated regional multidisciplinary team (MDT) meeting from 2005 to 2014. While there is no formal radiological definition of a biliary mass or stricture, for the purposes of this study, we used any finding on appropriate cross-sectional imaging where, after expert gastrointestinal radiologist review, there was significant concern for presence of a soft-tissue abnormality, including sufficient change in caliber of biliary duct with evidence of proximal dilation/obstruction, to justify further invasive investigation. These included extrahepatic and perihilar CCAs. Only patients who were potentially suitable for surgical or oncological intervention were referred. EUS was performed prior to ERCP wherever possible to reduce impact on staging accuracy [14], unless the local referring hospitals felt there was an urgent clinical need for stenting in highly symptomatic patients. If positive cytology had already been obtained from biliary brushings, allowing a definitive diagnosis, patients were not referred for EUS-FNA. Other exclusion criteria included those in whom FNA was not undertaken since EUS did not demonstrate a thickening or mass, or in whom the distal stricture was caused by a pancreatic mass. Written informed consent was obtained from all patients prior to EUS.

\section{EUS-FNA technique}

EUS was performed with either the Olympus GF-UC140P (Olympus Corp) or Pentax 32-UA (Pentax Precision Instruments, Orangeburg, New York, United States) linear-array echoendoscopes, under conscious sedation (using midazolam and fentanyl) by one of three expert endoscopists, all with experience of over 2000 procedures.

The department's FNA technique has been previously described [15]. The EUS-FNA was performed utilizing a 22 gauge or 25G FNA needle (Boston Scientific 'Expect,' Cook Medical 'Echo 3' or 25G Boston Scientific 'Expect') depending on the position and degree of flexion of the echoendoscope as judged by the echoendoscopist during the procedure. For each pass, the needle was moved back and forth through the lesion for a mean of 10 movements under suction pressure applied by a $10-\mathrm{mL}$ syringe set at $5 \mathrm{~mL}$. If excessive blood was deemed to be present in the initial sample, acquisition with no suction was substituted. The needle was placed under the surface of neutral buffered formalin solution and the sample gently extruded with the stylet to produce a visible strand of tissue for direct histological processing. A minimum of three passes and a maximum of six passes (if an inadequate sample was noted in the formalin solution) of the needle were performed. The sample was then filtered through a micro-cassette and then transferred for standard histological processing. We did not perform smear or liquid cytology. Rapid on-site evaluation (ROSE) was not used.

\section{Recording of clinical, radiological and outcome data}

Clinical and radiological information was prospectively recorded immediately following the procedure, with data points including mass vs stricture ( $\triangleright$ Fig. 1 ) and presence of stent and position. FNA results were added when available. Outcome data were based upon histology of resection specimens in those who underwent surgery, or on clinical status after a minimum of 4 years of follow-up through the MDT in those who did not undergo surgery. Lesions were classified as benign if there was no evidence of progression on serial imaging at most recent clinic review.

\section{Cytopathological assessment}

The specimen was assessed by one experienced cytologist/histopathologist and graded based on the Papanicolaou Society of Cytopathology nomenclature, which was proposed in an effort to reduce inter-observer variability and improve diagnostic reproducibility [16]. Results were classified into five categories: 1 

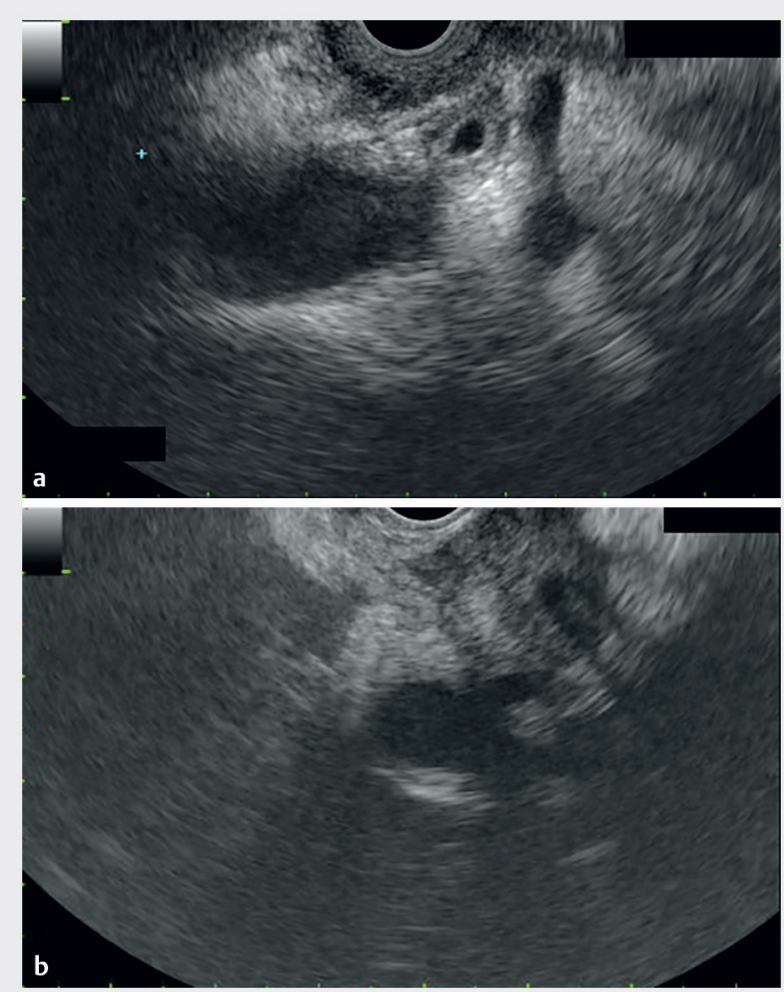

- Fig. 1 EUS images showing a bile duct thickening and $\mathbf{b}$ bile duct mass.

to 5 of "non-diagnostic," “negative," "atypical," "suspicious for malignancy," or "positive," based on the findings of increased cellularity, multiple single atypical cells, loss of honeycomb arrangement, increased nuclear to cytoplasmic ratio, loss of polarity, cell-in-cell arrangement, nuclear molding, chromatin clumping, and irregular nuclear membrane ( $>$ Fig. 2). Further analysis was performed with varying degrees of cytological suspicion. These grades are showing increasing acceptance, but have not been well validated in the clinical setting. We performed initial analysis with only those findings in the unequivocal positive class, but evaluated test performance of using lower thresholds for considering a sample positive, in an effort to validate this scoring approach clinically.

\section{Ethical approval}

EUS data were captured prospectively as part of a clinical audit program with institutional approval. Linkage to clinical data was subsequently performed for the purposes of this study under ethical approval obtained from the NHS Research Ethics Service Committee North East in March 2015.

\section{Data analysis}

Data on EUS-FNA findings, histology and patient outcomes were combined with other patient data for descriptive analysis, including analysis of test performance. All statistical tests were performed using $R$ (version 3.1.2) [17]. The Fisher's exact test was performed to assess the statistical significance of differen-
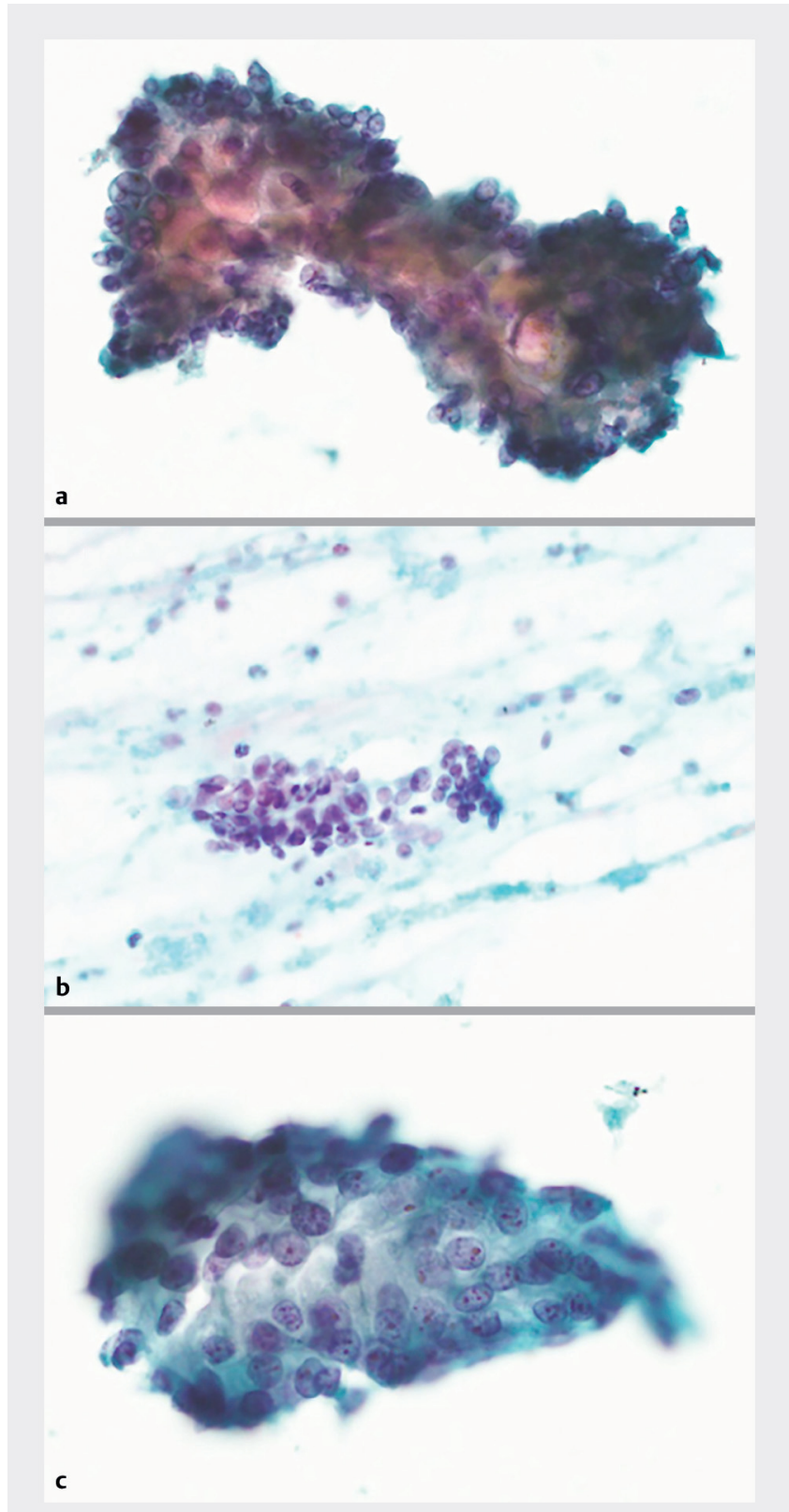

- Fig. 2 Cytological features of bile duct EUS-FNA specimens. a Cells with large pleomorphic nuclei, prominent nucleoli, crowding and overlapping. Diagnosis: malignant (Score 5). b Cells with some pleomorphism and small nucleoli. There is nuclear crowding and overlapping. Background acute inflammation suggestive of reactive atypia. Diagnosis: Inconclusive-favoring benign (Score 3). c Cells with moderately large pleomorphic nuclei and small nucleoli, focal crowding and overlapping. Diagnosis: Inconclusive-favoring malignant (Score 4). Cytological features in bile duct FNA specimens lie on a continuum from "benign" to "malignant." Unfortunately, many samples have features near the center of this continuum, and are "inconclusive." There are currently no biomarkers in clinical use to assist in the diagnosis of inconclusive FNA samples.

ces in test sensitivity. Patient and endoscopic factors potentially predicting an inaccurate diagnostic result were analyzed using multiple linear regression. Stepwise model selection was 
performed by optimization of the Aikake information criteria (AIC). A two-tailed $P<0.05$ was defined as statistically significant for all analyses.

\section{Results}

\section{Patient population}

Ninety-seven patients were included in the analysis ( $54 \%$ male, median age 68 years, range $26-93$ years). Thirteen patients had a known history of primary sclerosing cholangitis (PSC) diagnosed prior to MDT referral using appropriate clinical investigations in our tertiary center liver clinic. Due to concerns around the NPV of a benign EUS-FNA result, 13 patients underwent more than one procedure, including two patients who each underwent three procedures after an initial or repeat sample was judged to be non-diagnostic. This resulted in a total of 112 EUSFNA procedures included in the analysis. The key patient characteristics and EUS findings are summarized in $>$ Table 1. All patients were followed to the point of definitive surgical diagnosis or for at least four years if no operation was performed (range 48-168 months). No patients were lost to follow-up. There were no reported adverse events from EUS-FNA in this study.

Eighty of 97 patients had confirmed malignancy following surgical resection, resulting in a prevalence of cholangiocarcinoma in our population of $82.5 \%$. Four of 112 samples (3.6\%) were reported by the histopathologist as inadequate for diagnostic purposes and were excluded from subsequent analysis.

\section{Test performance}

Definitive outcome data were based upon surgical histopathology in those undergoing resection or upon outcome data after a minimum of 4 years of follow-up. Those patients alive, symptom-free, and with non-progressive imaging at the latest point of follow-up were presumed to have non-malignant pathology. Overall test sensitivity for the 108 EUS-FNA procedures that yielded an adequate sample was $75 \%$ (95\% Cl 64\%-84\%), with a specificity of $100 \%(95 \% \mathrm{Cl} 84 \%-100 \%)$. This resulted in a NPV of 0.62 (95\% Cl 0.47-0.75).

We used multiple logistic regression analysis to model predictors of obtaining an inaccurate diagnostic result. Lesions positioned at the hilum (association coefficient $-1.53, P=0.026$ ), the presence of a biliary stent (association coefficient $-1.95, P=$ 0.004 ) and diagnosis of PSC (association coefficient-1.53, $P=$ 0.026 ) were significantly independently associated with an inaccurate result ( $\downarrow$ Table 2 ). Similarly, the odds ratios (OR) for the likelihood of an accurate test result in cases of hilar lesions or in the presence of a biliary stent or in patients with a diagnosis of PSC were 0.14 (95\% Cl 0.03-0.50), 0.16 (95\% Cl 0.02$0.99)$ and $0.22(95 \% \mathrm{Cl} 0.05-0.79)$ respectively. Other clinical factors not significantly predictive of result accuracy included lesion size, lymph node involvement, presence of gallstones and gauge of needle used.

Although test specificity was not affected (remaining 100\% in all subsets), in the subset of patients without presence of a stent, the sensitivity of EUS-FNA improved considerably to 91 $\%$; in those patients with neither PSC nor a stent and with lesions away from the hilum (i.e. distal CBD), sensitivity in-
- Table 1 Key patient characteristics and EUS findings.

\begin{tabular}{|l|l|}
\hline Total number of patients & 97 \\
\hline Median age (range) & $68 \mathrm{yr}(26-93 \mathrm{yr})$ \\
\hline Gender (male, female) & $54 \%, 46 \%$ \\
\hline Known history of PSC & $13 \%$ \\
\hline Stricture appearance & $\begin{array}{l}44 \% \text { thickening } \\
56 \% \text { mass }\end{array}$ \\
\hline Median mass size (range) & $1.3 \mathrm{~cm}(0.7-4 \mathrm{~cm})$ \\
\hline Location & $\begin{array}{l}46 \% \text { Hilar/Hepatic duct } \\
56 \% \text { Distal/CBD }\end{array}$ \\
\hline Presence of biliary stent & $46 \%$ \\
\hline Presence of abnormal lymph nodes & $40 \%$ \\
\hline Presence of gallstones & $18 \%$ \\
\hline Needle size & 22 gauge-39\% \\
\hline Number of malignancy-confirmed cases & 80 gauge-61\% \\
\hline (percentage) & \\
\hline
\end{tabular}

PSC, primary sclerosing cholangitis; CBD, common bile duct

- Table 2 Multiple logistic regression analysis of predictors for inaccurate diagnostic result for cholangiocarcinoma using EUS-FNA

\begin{tabular}{|l|l|l|l|}
\hline Factor & Coefficient & $\begin{array}{l}\text { Odds Ratio for } \\
\text { Accurate Result } \\
(\mathbf{9 5} \% \mathbf{C I})\end{array}$ & P value \\
\hline Presence of stent & -1.95 & $\begin{array}{l}0.14 \\
(0.03-0.50)\end{array}$ & 0.004 \\
\hline Diagnosis of PSC & -1.86 & $\begin{array}{l}0.16 \\
(0.02-0.99)\end{array}$ & 0.049 \\
\hline Hilar position & -1.53 & $\begin{array}{l}0.22 \\
(0.05-0.79)\end{array}$ & 0.026 \\
\hline
\end{tabular}

EUS, endoscopic ultrasound-guided fine-need aspiration; PSC, primary sclerosing cholangitis

creased further to $95 \%$, with a NPV of 0.93 . In contrast, in those patients without PSC in whom a stent had been placed, sensitivity dropped to $65 \%$, while for the subgroup of patients in this group with a hilar lesion, sensitivity decreased further to $56 \%$ ( Table 3 ).

\section{Utility of repeat sampling}

The total dataset included samples from 13 patients undergoing more than one procedure due to ongoing concerns expressed by the MDT after a reportedly non-malignant initial histological sample. This included one patient in whom the initial histology report of severe dysplasia was changed after expert review to definite malignancy, but only after a repeat EUS-FNA had been performed. We analyzed the test performance after the first EUS-FNA procedure in the whole cohort (i.e. both those who underwent just a single EUS procedure and those 
Table 3 Diagnostic performance of model in non-PSC patient subsets.

\begin{tabular}{|l|l|l|l|l|}
\hline & Stent & \multicolumn{2}{|c|}{ No Stent } & \\
\hline Location of lesion & Sensitivity & NPV & Sensitivity & NPV \\
\hline Hilar & $56 \%$ & 0.33 & $86 \%$ & 0.40 \\
\hline Non-hilar (distal CBD) & $65 \%$ & 0.67 & $95 \%$ & 0.93 \\
\hline
\end{tabular}

NPV, negative predictive value; PSC, primary sclerosing cholangitis; $\mathrm{CBD}$, common bile duct

that underwent more than one EUS procedure) and obtained similar performance characteristics to that previously described ( $\downarrow$ Table 4 ). In the subset of 13 patients who underwent more than one EUS-FNA due to ongoing clinical concerns, performance after the first EUS procedure was much poorer, with an overall sensitivity of just $17 \%$ ( Table 4 ). Repeat sampling in these patients improved test performance, but sensitivity remained low at $50 \%$ ( $\triangleright$ Table 4 ).

\section{Use of graded histological classification}

In the absence of clear evidence of invasive cancer, histopathological assessment can nonetheless be stratified by the degree of cellular atypia observed. An expert cytopathologist used a discrete scale from 1 to 5 ( $\triangleright$ Fig. 2) based on the previously described scoring system to denote the degree of atypia observed. This classifies results into five grades: 1 to 5 of "non-diagnostic," “negative," “atypical/favoring benign," "suspicious for malignancy," or "positive" based on the findings of increased cellularity, multiple single atypical cells, loss of honeycomb arrangement, increased nuclear to cytoplasmic ratio, loss of polarity, cell-in-cell arrangement, nuclear molding, chromatin clumping, and irregular nuclear membrane. In the original MDT discussions, samples were considered positive only in the presence of clear histopathological evidence of malignancy, corresponding to a score of 5. Samples corresponding to lower scores suggestive of degrees of atypia were not considered positive or more formally stratified. We retrospectively analyzed test performance using various formal thresholds of this cytopathological score to define test positivity. Using a threshold of a score of $\geq 4$, overall test performance for an initial EUS-FNA improved from a sensitivity of $75 \%$ to $83 \%$ without any loss of specificity. This corresponded to a NPV improvement from 0.62 to 0.70 . Restricting our analysis to the first EUS-FNA procedure undergone by all patients showed similar results ( $>$ Table 4). In those 13 patients who underwent more than one EUSFNA due to clinical suspicion, test sensitivity using the aforementioned cytopathology score threshold of $\geq 4$ remained extremely poor at $33 \%$ after the index procedure.

However, after the second EUS procedure, combining data from both procedures and using a cytopathology score threshold of $\geq 4$ led to a statistically significant improvement in sensitivity from $17 \%$ (following the first EUS using a cytopathology score threshold of 5) to $100 \%(P=0.015)$. Importantly, there was no loss of specificity, which remained at $100 \%$. The corresponding improvement in NPV in this challenging diagnostic

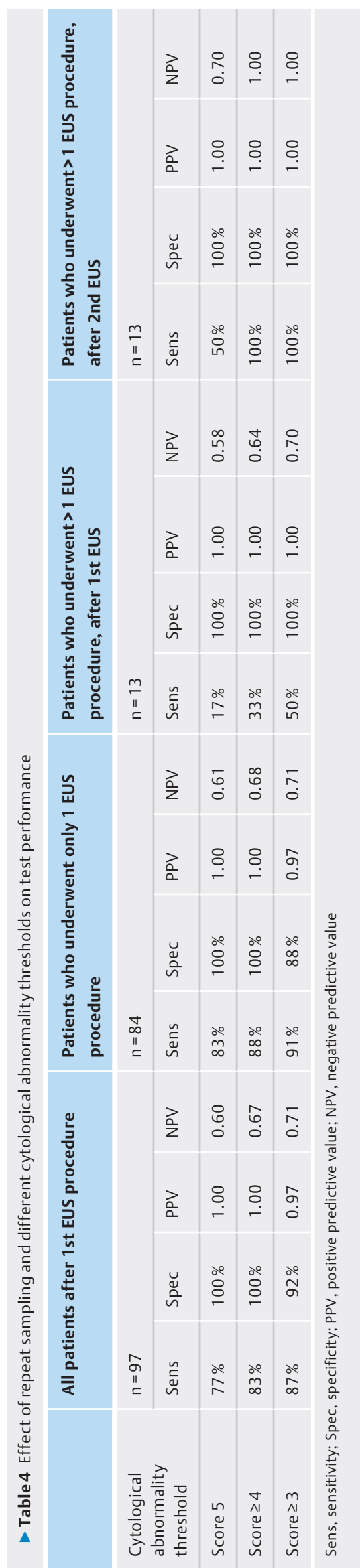


group was from 0.58 after a single procedure with standard histological scoring, to 1.00 after two procedures using the alternative scoring system with a threshold of $\geq 4$. Analysis by patient subgroup revealed markedly improved test performance in the group of non-PSC patients previously identified as having adverse predictive factors in the form of a biliary stent and/or a hilar lesion (Supplemental Table 1).

\section{Discussion}

Tissue confirmation and accurate staging are vital in preoperative management of biliary strictures and EUS remains the most accurate test for this [18]. However, in the limited number of retrospective and prospective published case series on EUSFNA, test NPV has consistently been demonstrated to be poor $[10,19]$. Furthermore, the small numbers of patients included in previous studies have limited analysis, hampering our understanding of test performance in various clinical situations [5, 19-21]. The resulting lack of confidence in test performance informs surgical decision making and has resulted in rates of inappropriate, highly morbid surgery of up to $20 \%$, even in centers where EUS-FNA usage is well established. This is the largest prospective case series to date of EUS-FNA of biliary lesions, all managed through a single regional MDT with standardized care and decision making, and with no loss to follow-up, i.e. until surgery or a minimum of $\mathbf{4 8}$ months in those patients not undergoing an operation.

Assessment of our overall test performance demonstrated a sensitivity of $75 \%$ and specificity of $100 \%$. Adequate samples for diagnostic purposes were obtained in all except four cases, giving a $3.6 \%$ inadequate sampling rate in line with previously reported rates of between $2 \%$ to $5 \%$ [20,22-24]. Importantly, because our center EUS-FNA is reserved for clinically challenging cases, our patient group was by definition difficult to assess. This sensitivity is in line with published studies of EUSFNA of between $47 \%$ to $89 \%[5,10,19-21,25]$ and is noticeably better than sensitivities found at ERCP of $9 \%$ to $58 \%$, which has traditionally been the mainstay for diagnosis $[10-12,19]$. The specificity is clearly superior to ERCP at $61 \%$ to $100 \%$, as well as MRCP, which cannot reliably distinguish benign from malignant strictures [26].

In the largest previous published case series by DeWitt et al [10], the NPV was only 0.29 , and a meta-analysis demonstrated a pooled negative likelihood ratio of 0.26 [27]. In our series, the NPV was noticeably higher at 0.62 ; however, across the entire cohort, this clearly remains too poor to adequately reassure a surgeon that malignancy has been excluded. Nevertheless, within our large sample, we were able to identify factors predictive of improved test performance which will help operators understand circumstances when test reliability might be compromised.

In particular, the effect on EUS-FNA accuracy due to presence of a stent has not previously been demonstrated in biliary lesions, with no statistically significant difference found in the sensitivity of stented versus unstented in any study. However, there has been a suggestion that a stent may limit access to malignant tissue on the distal side [25]. We found that presence of a stent reduced the likelihood of an accurate test result, with an OR of 0.14 (95\% Cl $0.03-0.50)$. This is unlikely to represent a mechanical sampling problem, but more likely due to the effect on cytology, since an inflammatory response in the presence of a biliary stent can produce cytologic changes mirroring atypia (Supplemental Fig.1). This further reinforces the message of the America Society of Gastrointestinal Endoscopy (ASGE) guidelines that EUS should be performed before stenting whenever feasible and clinically safe to do so, unless evidence of acute cholangitis or severe pruritis. Nonetheless, $46 \%$ of patients in our series had undergone prior placement of a stent, demonstrating that this message has not yet found widespread acceptance into practice.

A recent meta-analysis examining lesion location found a higher sensitivity for distal strictures compared with proximal, although concluding the impact on accuracy should be further assessed in future studies due to the low number of relevant studies resulting in wide confidence intervals [27]. We found a statistically significant outcome, with an OR for an accurate result for a hilar lesion 0.22 (95\% Cl 0.05-0.79) when compared with the CBD. This is likely due to the more difficult endoscopic access to these lesions, with the hilum a greater distance from the transducer. Poor penetration of ultrasound into the hilum for more proximal lesions may obscure the precise outline of the lesion and make FNA more difficult to target. The performance of EUS ultrasound technology has steadily improved and full delineation of hilar structures may now be considerably easier with more modern equipment.

We also demonstrated that a prior diagnosis of PSC reduced test accuracy (OR 0.16, $95 \% \mathrm{Cl} 0.02-0.99$ ); this has been previously suggested in a result failing to achieve statistical significance [28]. We postulate that this is due to presence of inflammation and atypia. Use of ursodeoxycholic acid has shown some efficacy in improving biochemical abnormalities and stabilizing hepatic inflammation [29, 30] and could be considered to improve diagnostic accuracy, although this remains purely speculative.

A number of factors were not statistically significantly predictive of test performance in our multivariate analysis. These included FNA needle gauge. This knowledge is useful, as the duodenal position often hampers the ability to use larger needles.

Taken together, our data suggest that for patients with distal lesions, without PSC or a stent, overall test sensitivity and NPV are excellent ( $\triangleright$ Table 3 ). Although in this subgroup a benign result at EUS can be relied on to exclude cancer, this combination occurred in only $30 \%$ of patients in our series, meaning that for most patients, concerns will remain regarding test performance.

Our analysis also demonstrated that consideration of samples showing atypical features that fell short of overt malignancy (score $\geq 4$ ) increased test performance ( Table 4 ). This was particularly useful in those patients with features identified as predictive of reduced test performance i.e. presence of stent and hilar lesion (Supplemental Table 1). Importantly, along with an increase in sensitivity there was no loss of specificity at 
this threshold, meaning that patients with benign pathology would not be put forward for unnecessary surgery.

In a subset of patients where clinical concerns were raised despite an apparently reassuring initial EUS-FNA result, repeat sampling somewhat improved test performance, but the NPV still remained 0.70; a level that would fail to reassure most ( $\triangleright$ Table 4 ). In these most challenging cases facing our MDT, the combination of repeat sampling with a cytological threshold score of 4, markedly improved test sensitivity from $17 \%$ to $100 \%$ ( $\triangleright$ Table 4). Furthermore, if two samples were taken, and neither showed any concerning atypia (cytologic score $<4$ ), then the NPV was 1.00, and hence these patients could be safely monitored and avoid any unnecessary surgery.

It is worth noting the theoretical risk of peritoneal seeding with transperitoneal FNA. Several studies, however, have reassuringly demonstrated that EUS-FNA is not associated with an increased rate of peritoneal seeding or mortality in patients with pancreatic cancer [31-33] or CCA [34]. While the theoretical risk means trans-peritoneal FNA currently precludes a patient with hilar CCA from being eligible for liver transplantation in the United States [35], elsewhere EUS-FNA remains the method of choice for the diagnostic workup of high-risk biliary and pancreatic lesions [36].

Our MDT practice was to refer patients for EUS-FNA where this could inform clinical management. Thus, patients who were obviously not suitable for surgical or oncological intervention were not referred and were not captured in this study. This limitation might affect reported test accuracy, though we note that our findings therefore reflect test performance under "real-world" conditions. Other potential limitations, are that we did not collect data on the use of plastic versus metal stents, or the numbers of patients who underwent EUS-FNA after nondiagnostic biliary brushings on ERCP, and hence could not analyze the impact of these separately. Finally, the number of patients who underwent repeat EUS-FNA in our cohort was small, even though we were able to demonstrate meaningful results. A future study with a larger patient cohort would be recommended to validate these findings.

\section{Conclusion}

In summary, we report a prospective case series of EUS-FNA of biliary lesions which was adequately powered to allow the identification of clinical factors that can help guide the MDT in interpretation of test performance. In addition, in the form of repeat procedures and/or use of a standardized cytological scoring system, we describe measures to improve test performance in even the most challenging patient groups to levels that will allow accurate selection of patients who might benefit from cancer surgery, while affording reassurance to patients and clinicians alike in those deemed to have non-malignant pathology. In the future, additional markers should be sought to differentiate benign from malignant atypia on cytology and reduce the need for repeat samples.
Competing interests

The authors declare that they have no conflict of interest.

\section{References}

[1] Patel T. Increasing incidence and mortality of primary intrahepatic cholangiocarcinoma in the United States. Hepatology 2001; 33: 1353-1357

[2] Taylor-Robinson SD, Toledano MB, Arora S et al. Increase in mortality rates from intrahepatic cholangiocarcinoma in England and Wales 1968-1998. Gut 2001; 48: 816-820

[3] Patel AH, Harnois DM, Klee GG et al. The utility of CA 19-9 in the diagnoses of cholangiocarcinoma in patients without primary sclerosing cholangitis. Am J Gastroenterol 2000; 95: 204-207

[4] Clayton RAE, Clarke DL, Currie EJ et al. Incidence of benign pathology in patients undergoing hepatic resection for suspected malignancy. Surgeon 2003; 1: 32-38

[5] Fritscher-Ravens A, Broering DC, Knoefel WT et al. EUS-guided fineneedle aspiration of suspected hilar cholangiocarcinoma in potentially operable patients with negative brush cytology. Am J Gastroenterol 2004; 99: 45-51

[6] Gerhards MF, Vos P, van Gulik TM et al. Incidence of benign lesions in patients resected for suspicious hilar obstruction. Br J Surg 2001; 88: $48-51$

[7] Uhlmann D, Wiedmann M, Schmidt F et al. Management and outcome in patients with Klatskin-mimicking lesions of the biliary tree. J Gastrointest Surg 2006; 10: 1144-1150

[8] Nakayama A, Imamura H, Shimada R et al. Proximal bile duct stricture disguised as malignant neoplasm. Surgery 1999; 125: 514-521

[9] Wetter LA, Ring E], Pellegrini CA et al. Differential diagnosis of sclerosing cholangiocarcinomas of the common hepatic duct (Klatskin tumors). Am J Surg 1991; 161: 57-62; discussion 62-63

[10] DeWitt J, Misra VL, LeBlanc JK et al. EUS-guided FNA of proximal biliary strictures after negative ERCP brush cytology results. Gastrointest Endosc 2006; 64: 325-333

[11] Selvaggi SM. Biliary brushing cytology. Cytopathology 2004; 15: 7479

[12] Khashab MA, Fockens P, Al-Haddad MA. Utility of EUS in patients with indeterminate biliary strictures and suspected extrahepatic cholangiocarcinoma (with videos). Gastrointest Endosc 2012; 76: 10241033

[13] Hammoud GM, Almashhrawi A, Ibdah JA. Usefulness of endoscopic ultrasound-guided fine needle aspiration in the diagnosis of hepatic, gallbladder and biliary tract Lesions. World J Gastrointest Oncol 2014; 6: 420-429

[14] Cannon ME, Carpenter SL, Elta GH et al. EUS compared with CT, magnetic resonance imaging, and angiography and the influence of biliary stenting on staging accuracy of ampullary neoplasms. Gastrointest Endosc 1999; 50: 27-33

[15] Brais RJ, Davies SE, O’Donovan M et al. Direct histological processing of EUS biopsies enables rapid molecular biomarker analysis for interventional pancreatic cancer trials. Pancreatology 2012; 12: 8-15

[16] Pitman MB, Layfield LJ. Guidelines for pancreaticobiliary cytology from the Papanicolaou Society of Cytopathology: A review. Cancer Cytopathol 2014; 122: 399-411

[17] R Core Team. R: A language and environment for statistical computing. R Foundation for Statistical Computing; 2014: Available at: https://www.R-project.org/ 
[18] Baron TH, Mallery JS, Hirota WK et al. The role of endoscopy in the evaluation and treatment of patients with pancreaticobiliary malignancy. Gastrointest Endosc 2003; 58: 643-649

[19] Eloubeidi MA, Chen VK, Jhala NC et al. Endoscopic ultrasound-guided fine needle aspiration biopsy of suspected cholangiocarcinoma. Clin Gastroenterol Hepatol 2004; 2: 209-213

[20] Meara RS, Jhala D, Eloubeidi MA et al. Endoscopic ultrasound-guided FNA biopsy of bile duct and gallbladder: analysis of 53 cases. Cytopathology 2006; 17: 42-49

[21] Lee JH, Salem R, Aslanian $H$ et al. Endoscopic ultrasound and fineneedle aspiration of unexplained bile duct strictures. Am J Gastroenterol 2004; 99: 1069-1073

[22] Williams DB, Sahai AV, Aabakken L et al. Endoscopic ultrasound guided fine needle aspiration biopsy: a large single centre experience. Gut 1999; 44: 720-726

[23] Fritscher-Ravens A, Broering DC, Sriram PV et al. EUS-guided fineneedle aspiration cytodiagnosis of hilar cholangiocarcinoma: a case series. Gastrointest Endosc 2000; 52: 534-540

[24] Ranney N, Phadnis M, Trevino J et al. Impact of biliary stents on EUSguided FNA of pancreatic mass lesions. Gastrointest Endosc 2012; 76 : 76-83

[25] Mohamadnejad M, DeWitt JM, Sherman S et al. Role of EUS for preoperative evaluation of cholangiocarcinoma: a large single-center experience. Gastrointest Endosc 2011; 73: 71-78

[26] Sai JK, Suyama M, Kubokawa Y et al. Early detection of extrahepatic bile-duct carcinomas in the nonicteric stage by using MRCP followed by EUS. Gastrointest Endosc 2009; 70: 29-36

[27] Sadeghi A, Mohamadnejad M, Islami F et al. Diagnostic yield of EUSguided FNA for malignant biliary stricture: a systematic review and meta-analysis. Gastrointest Endosc 2016; 83: 290-298.e1
[28] Levy M], Baron TH, Clayton AC et al. Prospective evaluation of advanced molecular markers and imaging techniques in patients with indeterminate bile duct strictures. Am J Gastroenterol 2008; 103: 1263-1273

[29] Poropat G, Giljaca V, Stimac D et al. Bile acids for primary sclerosing cholangitis.Cochrane Database of Systematic Reviews. Chichester, UK: John Wiley \& Sons, Ltd; 2011

[30] Shi ], Li Z, Zeng X et al. Ursodeoxycholic acid in primary sclerosing cholangitis: meta-analysis of randomized controlled trials. Hepatol Res 2009; 39: 865-873

[31] Micames C, Jowell PS, White R et al. Lower frequency of peritonea carcinomatosis in patients with pancreatic cancer diagnosed by EUSguided FNA vs. percutaneous FNA. Gastrointest Endosc 2003; 58: 690-695

[32] Ikezawa K, Uehara H, Sakai A et al. Risk of peritoneal carcinomatosis by endoscopic ultrasound-guided fine needle aspiration for pancreatic cancer. J Gastroenterol 2013; 48: 966-972

[33] Kim SH, Woo YS, Lee KH et al. Preoperative EUS-guided FNA: effects on peritoneal recurrence and survival in patients with pancreatic cancer. Gastrointest Endosc 2018; 88: 926-934

[34] El Chafic AH, DeWitt JM, Sherman S et al. Mo1375 preoperative endoscopic ultrasound-fine needle aspiration (EUS-FNA): impact on long-term outcomes for cholangiocarcinoma (CCA). Gastrointest Endosc 2011; 73: AB323-AB324

[35] Gleeson FC, Lee JH, Dewitt JM. Tumor seeding associated with selected gastrointestinal endoscopic interventions. Clin Gastroenterol Hepatol 2018; 16: 1385-1388

[36] Chapman MH, Thorburn D, Hirschfield GM et al. British Society of Gastroenterology and UK-PSC guidelines for the diagnosis and management of primary sclerosing cholangitis. Gut 2019; 68: 1356-1378 